\title{
A HF/CI-SD Study of the Low-Lying States of Nitroprusside Ion
}

\author{
Eduardo Hollauer*a, and José A. Olabe ${ }^{b}$ \\ ${ }^{a}$ Universidade Federal Fluminense, Instituto de Química, Departamento de Físico- \\ Química, Morro do Valonguinho s/n, Centro, \\ 24210-150 Niterói - RJ, Brazil; e-mail gfqholl@vm.uff.br \\ ${ }^{b}$ Departamento de Química Inórganica, Analítica y Química Física (Inquimae), \\ Faculdad de Ciencias Exactas y Naturales, Universidad de Buenos Aires, Pabellón 2, \\ Ciudad Universitaria, Capital Federal 1428, Argentina; \\ e-mail olabe@ayelen.q3.fcen.uba.ar
}

Received: August 23, 1996

Desde a descoberta de dois estado meta-estáveis gerados por excitação ótica do nitroprussiato de sódio cristalino, $\mathrm{Na}_{2}\left[\mathrm{Fe}(\mathrm{CN})_{5} \mathrm{NO}\right] .2 \mathrm{H}_{2} \mathrm{O}$ (SNP), exibindo longa meia-vida a temperaturas abaixo de $160 \mathrm{~K}$, grande esforço vem sendo realizado no sentido de entender a estrutura eletrônica de sistemas correlatos. Apesar deste esforço, a natureza dos orbitais de fronteira e dos processos de excitação eletrônica de baixa energia são, ainda hoje, controversos. Cálculos iniciais EHT mostram que o HOMO possuia caráter do orbital $3 d$ metálico, enquanto o LUMO possuia uma contribuição dominantemente $\pi^{*}$ (NO). Entretanto, cálculos INDO definiram o orbital metálido $d$ como orbital de valência interna com vários elétron-volts em energia de Koopmans. O espectro vertical foi estimado através de cálculos $\mathrm{HF} / \mathrm{Cl}-\mathrm{SD}$ com bases de qualidade double-zeta e potenciais de caroço. As energias de Koopmans para os resultados ab initio estão de acordo com os resultados de Bottomley e Grein, e atribuem as primeiras excitações a processos de transferência de carga (TC) do trans-cyano para os ligantes nitrosila. Forças de oscilador foram calculadas e os resultados mostraram intensidades comparáveis com os resultados experimentais. A energia de excitação para o processo de transferência de carga metal $\rightarrow N O, 8 e \rightarrow 13 e\left(d_{\mathrm{xz}}, d_{\mathrm{yz}} \rightarrow \pi^{*} \mathrm{NO}\right)$ foi estimada em $4.52 \mathrm{eV}$ e exibindo intensa banda de absorção. A segunda excitação de transferência de carga, $1 b_{2}$ $\rightarrow 13 e\left(d_{\mathrm{xy}} \rightarrow \pi^{*} \mathrm{NO}\right)$, apontada por trabalhos prévios como uma típica banda de transferência de carga (TC) mostra uma pequena intensidade em $5.04 \mathrm{eV}$. Nos cálculos foi observado que o ordenamento SCF é muito independente do conjunto de base utilizado para o metal. Bases metálicas de caráter mínimo mostram resultados em grande concordância com os resultados EHT, enquanto as bases double-zeta empurram os orbitais $d$ metálicos para os orbitais de valência interna. $\mathrm{O}$ ordenamento das energias HF foi empregado no sentido de interpretar resultados de experimentos fotoquímicos e de termoanálise do SNP e os resultados parecem mostrar boa concordância com as propriedades calculadas.

Since the discovering of two photoexcited metastable states of crystalline sodium nitroprusside, $\mathrm{Na}_{2}\left[\mathrm{Fe}(\mathrm{CN})_{5} \mathrm{NO}\right] .2 \mathrm{H}_{2} \mathrm{O}(\mathrm{SNP})$, showing rather long lifetimes at temperatures below $160 \mathrm{~K}$, much effort has been devoted toward the study of its electronic structure. Despite this tremendous effort the nature of the frontier orbitals and the related low energy excitations remains controversial. Early calculations, EHT, showed the HOMO as mainly the metallic $3 d$ orbital while the LUMO had a major $\pi^{*}$ (NO) contribution. However INDO calculations, clearly set the metal $d$ orbital many electron-volts deep in core. The vertical electronic spectrum have been estimated through $a b$ initio HF/CI-SD with a double-zeta quality basis set. The ab initio results support Bottomley and Grein's interpretations and assign the first electronic transitions to ligand-to-ligand charge-transfer excitation from trans-cyano to nitrosyl ligands. The corresponding oscillator strengths have been calcu- 
lated showing comparable intensity with the experimental results. The excitation energy of the metal $\rightarrow N O$ charge-transfer transition, $8 e \rightarrow 13 e\left(d_{\mathrm{xz}}, d_{\mathrm{yz}} \rightarrow \pi^{*} \mathrm{NO}\right)$ have been estimated to be at $4.52 \mathrm{eV}$ and show a rather intense absorption band. The second CT excitation, $1 b_{2} \rightarrow 13 e\left(d_{\mathrm{xy}}\right.$ $\rightarrow \pi^{*} \mathrm{NO}$ ), pointed by previous works as a typical CT band, exhibits a small intensity at $5.04 \mathrm{eV}$. In the calculations it was observed that SCF orbital ordering are rather dependent on the metal basis set used. Metallic minimal basis set show results in close agreement with EHT early calculations while double-zeta basis set pushes the metallic $d$ orbitals deep away from the HOMO's. The HF orbital ordering has been used to interpret photochemical and thermoanalysis experiments on SNP and the results seem to fit properly with the calculated properties.

Keywords: ab initio (SCF,CI-SD), nitroprusside, excited states, metastable states, $\mathrm{Na}_{2}\left[\mathrm{Fe}(\mathrm{CN})_{5} \mathrm{NO}\right]$, pentacyanonitrosylferrate

\section{Introduction}

The disodium pentacyanonitrosylferrate (sodium nitroprusside), $\left.\mathrm{Na} 2 \mathrm{Fe}(\mathrm{CN})_{5} \mathrm{NO}\right] .2 \mathrm{H}_{2} \mathrm{O}$ (SNP) is a dark red crystal which structure is well known to be ortorhombic Pnnm with four molecules per unit cell ${ }^{1}$. The $\left[\mathrm{Fe}(\mathrm{CN})_{5} \mathrm{NO}^{-2}\right.$ anion shows an approximate $4 m$ local symmetry and the octahedra structure slightly distorts in the crystal with the angle $\mathrm{NC}-\mathrm{Fe}-\mathrm{NO}$ departing from linearity by about $3^{\circ}$. This complex shows a rather unusual $\mathrm{Fe}-\mathrm{NO}$ distance, $1.653 \AA$, and a slightly extended N-O distance, $1.124 \AA$, typical of a strong $N O \rightarrow$ metal $\sigma$ electron donation followed by intense metal $\rightarrow \mathrm{NO}$ back-donation. Another point of interest are the $\mathrm{Fe}-\mathrm{CN}$ distances that have nearly the same value for the equatorial (1.933 $\AA$; averaged; cis hereafter) and axial ligands (1.918 $\AA$; trans hereafter). For complexes of the type $\left[\mathrm{M}(\mathrm{CN})_{5} \mathrm{NO}^{-2}\right.$, where $\mathrm{M}$ is a metal of group VIII, the trans $\mathrm{Fe}-\mathrm{CN}$ distance is usually longer than cis distances. The trans $\mathrm{Fe}-\mathrm{CN}$ is nearly linear with the $\mathrm{Fe}-\mathrm{NO}$ bond but two possible orientations are found for the $\mathrm{Fe}-\mathrm{NO}$ bond in the crystal. They form an angle of $112^{\circ} 10^{\prime}$ in an almost anti-parallel orientation, both $\pm 33^{\circ} 55^{\prime}$ apart from a axis. Its UV-visible spectrum does not change qualitatively in solution supporting that environmental effects do not break the qualitative picture obtained from calculations on the isolated anion ${ }^{2}$.

Recently interest in elucidating the electronic structure of SNP has been reinforced by the discovery of similar long-living metastable states and to the increasing biological importance of this ion, kindly pointed by the referees. Following their suggestion is important to remark that nitroprusside is a drug, being presently commercialized in the USA under the name of Nipride, and takes important hole activating guanylyl cyclase, either via release of nitric oxide or via direct stimulation of the enzyme. The result is increased intracellular cBMP, which relaxes vascular smooth muscle by decreasing calcium levels in the cell. Nitroprusside acts as a vasodilator, decreases blood pressure and increases cardiac output. However, metabolism of nitroprusside results in the cyanide release, a very serious side effect. As such, nitroprusside is used parentally in treating hypertensive emergencies and severe cardiac failure.

Early calculations on the electronic structure of SNP have been carried out by Manoharan and Gray ${ }^{2}$ at SCCCEHT level. The ten highest occupied molecular orbitals (HOMO) obtained were $\left[\right.$ (core) $8 e^{4} 5 b_{1}^{2} 6 b_{1}^{2} 9 e^{4} 21 a_{1}^{2} 1 a_{2}^{2}$ $\left.10 e^{4} 11 e^{4} 12 e^{4} 2 b_{2}{ }^{2}\right]$ with the $2 b_{2}$ showing a major contribution of $3 d(x y)$ metal orbitals and the $13 e$ appearing as a mixture of $3 d(x z, y z)$ with the dominant contribution of $\pi^{*}$ NO. The average energy difference between the ten highest orbitals, is around $0.5 \mathrm{eV}$ with the two HOMO's lying within $0.56 \mathrm{eV}$, and apart by $2.3 \mathrm{eV}$ from the others in Koopmans' energy. Among the first virtual orbitals (LUMO) we find, in increasing energy order, $\left[13 e 7 b_{1} 22 a_{1}\right.$ $\left.14 e 23 a_{1}\right]$, well separated in energy $(\cong 4 \mathrm{eV})$ and with a HOMO-LUMO gap of around $2.54 \mathrm{eV}$. This pattern clearly indicates a spectrum dominantly governed by one-electron excitations from the two HOMO's to the first LUMO's. Based on this pattern Manoharan and Gray proposed the transition assignment presented in Table 1, for which the low energy excitations are clearly low-intensity CT transitions. These results were confirmed by Fenske and DeKock $^{3}$ with a modified CNDO method and other experimental works on the optical electronic spectrum, in solution and with polarized light in crystals ${ }^{4}$, ESR experiments $^{3}$, XPS experiments ${ }^{5}$ and by Mossbauer ${ }^{6}$ spectros- $^{-}$ copy claim these assignments to be correct.

However there exist evidences towards a different possibility. Chemical reactions of this complex with nucleophiles lead most often to substitution on nitrosyl despite the $\mathrm{Fe}-\mathrm{NO}$ distance is the shortest one in the complex. Photochemical studies do not support metal orbitals as the HOMO since irradiation on these bands show nitrosyl exchange rather than metal oxidation products. Partially based on these informations E. Wasielewska ${ }^{7}$ suggested that Manoharan ${ }^{2}$ assignment was inconsistent with some experimental information. The major inconsistency, fol- 
Table 1. Experimental excitation energies, intensities and proposed assignments for the eletronic spectrum of sodium nitroprusside.

\begin{tabular}{|c|c|c|c|c|c|}
\hline Band & energy $^{4}$ eV $\left(\lambda_{\mathrm{nm}}\right)$ & $\mathrm{SCCC}^{2}$ & SINDO $^{7,9}$ & $\varepsilon_{\max }^{4}$ & polariz. \\
\hline I & 2.49 (497) & $2 \mathrm{~b}_{2} \rightarrow 13 \mathrm{e}$ & $2 \mathrm{~b}_{2} \rightarrow 13 \mathrm{e}$ & 8 & $\perp$ \\
\hline II & $3.15(394)$ & $12 \mathrm{e} \rightarrow 13 \mathrm{e}$ & $12 \mathrm{e} \rightarrow 13 \mathrm{e}$ & 25 & | | \\
\hline III & $3.75(330)$ & $2 \mathrm{~b}_{2} \rightarrow 4 \mathrm{~b}_{1}$ & $12 \mathrm{e} \rightarrow 13 \mathrm{e}$ & 40 & $\perp$ \\
\hline IV & $4.68(265)$ & $12 \mathrm{e} \rightarrow 4 \mathrm{~b}_{1}$ & $1 \mathrm{a}_{2} \rightarrow 13 \mathrm{e}$ & 900 & - \\
\hline V & $5.20(258)$ & $11 \mathrm{e} \rightarrow 13 \mathrm{e}$ & & 700 & - \\
\hline
\end{tabular}

lowing Wasielewska, relates to the observed intensity in the low energy excitations of SNP, which showed rather small intensity transitions, something in disagreement with the usual intense and broad CT bands in similar compounds. A re-interpretation of thermal analysis of SNP, here presented, seems not to support metal orbitals as the HOMO.

Bottomley and Grein ${ }^{8}$ at New Brunswick and Golebiewski and Wasielewska ${ }^{7,9}$ at Cracow, independently carried out semi-empirical calculations for these transitions. The results are somehow conflicting but showed both, rather different results from the previous SCCC-EHT calculation. Carrying INDO calculations the first authors found a minor contribution of metal $d$ orbitals among the frontier orbitals. The results support the idea that $N O$ ligand strengths the trans-Fe- $\mathrm{CN}$ bond and the resulting orbital energy diagram shows the metallic $d$ orbitals too stable. Most of the occupied frontier orbitals are composed by trans and cis $\mathrm{CN}$ contributions. These authors claim the $\mathrm{CN}$ ligands role essentially as $\sigma$-donors while $\mathrm{NO}$ acts as a strong $\pi$-acceptor. As a consequence the first transitions are of a ligand-to-ligand type. According to SINDO calculations carried out by Golebiewski and Wasielewska ${ }^{7,9}$ however, the HOMO orbitals are of mixed character with about $40 \%$ of metal and $60 \%$ of cis-CN. All methods agree placing the $13 e$ orbital ( $\pi^{*} \mathrm{NO}$ ) as the first LUMO, the $3 b_{1}$ as a $3 d\left(x^{2}-y^{2}\right)$, and the $5 a_{1}$ as the $3 d\left(z^{2}\right)$. We report the SINDO assignments in Table 1 compared to the SCCC results.

Results obtained with SCCC, INDO and SINDO methods seem to provide rather different representations for the orbital diagram. Therefore the primary question in this study concerns the characterization of the nature of the involved orbitals.

Meanwhile this theoretical discussion was taking place, Mossbauer ${ }^{11}$ experiments indicated a metastable state generated through laser pumping in the $400-530 \mathrm{~nm}$ range at $160 \mathrm{~K}$. Below this temperature the metastable state is a long-living state which decays upon irradiation with red light. Violet light does not lead to depopulation of the metastable state. With longer wavelengths or at higher temperatures the metastable states cannot be populated. For a matter of convenience this state will be denoted as HMSNP (high-temperature metastable state nitroprusside). Fol- lowing Manoharan and Gray $^{2}$ this state have been assigned to the $2 b_{2}(x y) \rightarrow 13 e \pi^{*}$ (NO) orbital excitation. Due to the remarkable long life of this metastable state its Raman ${ }^{12,13}$ and IR spectrum ${ }^{15,16}$, polarized or not, optical UV-visible absorption spectra ${ }^{11,14}$, Mossbauer ${ }^{6}$ and ESR spectra have been obtained for many SNP salts. These experiments revealed the existence of a second metastable state below $90 \mathrm{~K}$, hereafter called as LMS-NP (low-temperature metastable state nitroprusside). ESR experiments do not support high-spin paramagnetism but after X-ray irradiation a doublet signal was attributed to the presence of $\left[\mathrm{Fe}(\mathrm{CN})_{5} \mathrm{NO}^{-3}\right.$. Based on these results the authors proposed the switching of the first LUMO's, $13 e$ and $22 a_{1}\left(d_{z} 2\right)$, one of the remaining few points in agreement over the several articles related to this study. Indeed this is consistent with the reduced $\mathrm{Fe}-\mathrm{NO}$ bending angle found in the metastable state.

Recently similar complexes have been found to exhibit long living electronic states. In the specific case of sodium nitroprusside, calorimetric and Raman experiments indicated LMS-NP and HMS-NP to lye 1.0 and $1.1 \mathrm{eV}$ above SNP. The authors point out the possibility of Jahn-Teller distortion caused the $12 e$ orbital splitting that would originate two possible metastable states. The intense depletion of metal $d_{x y}$ orbitals observed by Pressprich et al through $\mathrm{X}$-ray experiments favors the Manoharan-Gray energy levels but the significant increase of $d\left(z^{2}\right)$ metal population in the metastable state is difficult to understand under the same scheme.

Despite the tremendous effort in the generation of wide experimental information there is no clear understanding on the nature of these excited states. Following Terrile ${ }^{17}$ et al. the data obtained point out to some clear contradictions; a) The observed optical absorption for the first bands does not show an intensity consistent to a CT process and besides is not polarized along the charge flux as it should be; b) The observed trans effect leading to short trans $C N$ bonds by the NO presence should significantly change the $\mathrm{Fe}$-CN vibrational frequencies in the metastable state but this is not observed; c) The Mossbauer and ESR experiments indicate a possible exchange between the two LUMO of the system as well as to an increase of the metal dorbital population, something that is inconsistent with a CT excitation and all calculations reported so far; d) No splitting 
in the vibrational modes are observed; e) The metastable state X-ray spectra does not show appreciably bent $\mathrm{Fe}-\mathrm{NO}$ bonds, what is in clear conflict with the well establish nature of the first LUMO of the system.

Clearly there are a lot of misunderstanding and the calculation most of these results are based is a sixties EHT-SCCC calculation. M.R. Pressprich ${ }^{20}$ et al. reported an $a b$ initio calculation in close agreement with $\mathrm{INDO}^{8}$ results, that was judged as an unphysical result?!. Considering the wide successful results obtained with $a b$ initio methods in organic and inorganic compounds and the great controlability offered by this method is surprising how simply these results were discarded. Indeed many authors have been carring out HF/CI calculations on several metal transition compounds claiming an error smaller ${ }^{21,22}$ than $0.5 \mathrm{eV}$ to the experimental energies. The goal of this work, considering our limited computational resources, is to present simple, economical and balanced HF/CI-SD calculations designed to establish the correct qualitative orbital picture. The following section will present our results for SNP excitation energies while in the next some chemical evidences will be discussed.

\section{Calculations}

All the calculations performed employed an effective core potential (ECP) developed by Stevens, Jasien, Krauss and Basch $^{23}$ and a metal basis set containing four $s p$ shells plus three contracted $d$ functions [4sp,3d] suggested by Rappe, Smedley and Goddard ${ }^{24}$. With this ECP just ten metal electrons are removed from the calculations which keeps a good compromise between the deformation of the metal orbitals upon the bond formation and the computational cost. Care must be taken with $\mathrm{ECP}^{27}$ calculations when metal-ligand bond distances became too short due to the break of the central field approximation and our results have been checked upon this problem. For the ligands two basis sets were used. The first, for monitoring purposes only, was a minimal STO-3G basis set, hereafter called MNSL (minimal basis set for the ligands). The final results rely on Dunning' ${ }^{25}$ contraction of Huzinaga double zeta basis set, called through this article as DZSL (double-zeta basis set for the ligands). The total number of basis functions amounts 94 contracted gaussian functions for the MNSL and 142 basis functions for the DZSL. Due to the well-know tendency of ab-initio, as well when used with $\mathrm{ECP}^{2} \mathrm{~s}^{27}$, to generate extended geometries, all calculations employed DFT optimized geometry ${ }^{10}$ through a KohnSham SCF with gradient-corrected Becke functional. The geometry was properly symmetrized to $C_{4 v}$ geometry although all calculations employed the largest abelian subgroup, $C_{2 v}$. This geometry shows fairly good agreement with X-ray diffraction data and some important geometrical parameters used are listed below with the $X$-ray values between parenthesis; $\mathrm{Fe}-\mathrm{NO}$ distance of $1.65 \AA$ (1.653 $\AA$ ),
NO of $1.17 \AA$ (1.124 $\AA$ ), trans Fe-CN distance of $1.948 \AA$ $(1.918 \AA)$, trans $C N$ of $1.18 \AA(1.150 \AA)$, cis $\mathrm{Fe}-\mathrm{CN}$ distance of $1.948 \AA$ (1.933 $\AA$ ), cis $C N$ of $1.18 \AA$ (1.152 $\AA$ ). The $\mathrm{ON}-\mathrm{Fe}-\mathrm{CN}(\mathrm{cis})$ angle was set to $94.6^{\circ}$ but all others, due to the point group constrain, were set to $90^{\circ}$ or $180^{\circ}$.

Ground state Hartree-Fock calculation indicated a high density of states close to the HOMO-LUMO threshold. For the MNSL set it was found eleven orbitals within $2.8 \mathrm{eV}$ while for the DZSL these numbers are expanded to lye within $6.3 \mathrm{eV}$. Tables 2 and 3 present the Koopmans' energy and Mulliken orbital analysis for the basis set used. Both calculations indicate HOMO orbitals much closer in energy than expected from previous calculations. The ten upper energy levels determined in the DZSL calculation are [(core) $9 e^{4} 5 b_{1}^{2} 20 a_{1}^{2} 2 b_{2}^{2} 6 b_{1}^{2} 10 e^{4} 11 e^{4} 1 a_{2}^{2} 21 a_{1}^{2} 12 e^{4}$ ] while the six lowest energy unoccupied orbitals are defined as [13e 22a $\left.14 a_{1} 23 a_{1} 3 b_{2} 24 a_{1}\right]$ ]. The HOMO-LUMO Koopmans' energy gap have values around $8.8 \mathrm{eV}$ and the average energy spacing is 4 levels/eV for the occupied and around 0.55 levels/eV for the virtuals. A simple Mulliken analysis shows the metal d orbitals around 8.2-10.0 eV deep

Table 2. Koopmans' energy and Mulliken population analysis for the frontier orbitals. Calculations done with the MNSL basis. Interchanges with respect to the DZSL calculations are marked.

\begin{tabular}{lcl}
\hline Orbital & energy (eV) & \multicolumn{1}{c}{ Mulliken population } \\
\hline $3 \mathrm{~b}_{2}$ & 15.27 & $36 \% \mathrm{Fe} \mathrm{d}(\mathrm{xy}), 50 \%$ cis-CN \\
$23 \mathrm{a}_{1}$ & 13.54 & $58 \% \mathrm{Fe} 4 \mathrm{~s}, 46 \% \mathrm{Fe} \mathrm{p}_{\mathrm{z}}$ \\
$14 \mathrm{e}$ & 12.87 & $97 \% \mathrm{Fe}$ p's \\
$22 \mathrm{a}_{1}$ & 10.67 & $57 \% \mathrm{Fe} 4 \mathrm{~s}, 36 \% \mathrm{Fe} \mathrm{p}_{\mathrm{z}}$ \\
$13 \mathrm{e}$ & 8.74 & $16 \% \mathrm{~d}(\mathrm{xz}, \mathrm{yz}), 71 \% \pi *(\mathrm{NO})$ \\
$12 \mathrm{e}$ & -0.72 & $32 \%$ cis-CN, $64 \%$ trans-CN \\
$21 \mathrm{a}_{1}$ & -1.06 & $12 \% \mathrm{Fe}, 39 \%$ trans-CN, $48 \%$ cis-CN \\
$* 11 \mathrm{e}$ & -1.53 & $11 \% \mathrm{Fe}, 48 \%$ cis-CN, $22 \%$ trans-CN \\
$* 1 \mathrm{a}_{2}$ & -1.54 & $100 \%$ cis-CN \\
$10 \mathrm{e}$ & -1.61 & $11 \%$ metal, $89 \%$ cis-CN \\
$6 \mathrm{~b}_{1}$ & -1.93 & $99 \%$ cis-CN \\
$2 \mathrm{~b}_{2}$ & -2.15 & $15 \%$ metal, $85 \%$ cis-CN \\
$* 5 \mathrm{~b}_{1}$ & -2.77 & $22 \%$ of metal, $78 \%$ of cis-CN \\
$* 20 \mathrm{a}_{1}$ & -3.17 & $12 \%$ Fe, $42 \%$ cis-CN, $42 \%$ trans-CN \\
$9 \mathrm{e}$ & -3.38 & $15 \%$ of metal, $59 \%$ cis-CN \\
$19 \mathrm{a}_{1}$ & -3.86 & $10 \%$ metal, $81 \%$ cis-CN \\
$8 \mathrm{e}$ & -4.67 & $56 \%$ of metal d(xz, yz), $45 \% \mathrm{NO}$ \\
$18 \mathrm{a}_{1}$ & -6.02 & $16 \%$ of metal, $84 \%$ cis-CN \\
$1 \mathrm{~b}_{2}$ & -6.68 & $89 \%$ of metal d(xy) \\
\hline
\end{tabular}

Atomic Mulliken population.

-1.18 for $\mathrm{Fe},+0.12$ for trans- $\mathrm{C},-0.34$ for trans- $\mathrm{N},+0.13$ for cis- $\mathrm{C},-0.33$ for cis- $\mathrm{N},+0.21$ for $\mathrm{N}(\mathrm{NO}),-0.02$ for $\mathrm{O}(\mathrm{NO})$. 
Table 3. Koopmans' energy and Mulliken population analysis for the frontier orbitals. Calculation done with the DZSL basis.

\begin{tabular}{|c|c|c|}
\hline Orbital & energy $(\mathrm{eV})$ & Mulliken population \\
\hline $3 \mathrm{~b}_{2}$ & 11.56 & $15 \% \mathrm{Fe} \mathrm{d}_{\mathrm{xy}}, 85 \%$ cis-CN \\
\hline $23 \mathrm{a}_{1}$ & 11.43 & $59 \% \mathrm{Fe} 4 \mathrm{~s}, 40 \% \mathrm{Fe} \mathrm{p}_{\mathrm{z}}$ \\
\hline $14 \mathrm{e}$ & 10.14 & $98 \%$ Fe p's \\
\hline $22 \mathrm{a}_{1}$ & 8.74 & $30 \% \mathrm{Fe} 4 \mathrm{~s}, 70 \% \mathrm{Fe} \mathrm{p}_{\mathrm{z}}$ \\
\hline $13 \mathrm{e}$ & 4.87 & $16 \% \mathrm{~d}(\mathrm{xz}, \mathrm{yz}), 54 \% \pi^{*}(\mathrm{NO})$ \\
\hline $12 \mathrm{e}$ & -3.92 & $24 \%$ cis- $\mathrm{CN}, 75 \%$ trans- $\mathrm{CN}$ \\
\hline $21 \mathrm{a}_{1}$ & -4.57 & $9 \%$ metal, $22 \%$ trans- $\mathrm{CN}, 68 \%$ cis- $\mathrm{CN}$ \\
\hline $1 \mathrm{a}_{2}$ & -4.61 & $100 \%$ cis-CN \\
\hline $11 \mathrm{e}$ & -4.84 & $9 \%$ trans $-\mathrm{CN}, 86 \%$ cis- $\mathrm{CN}$ \\
\hline $10 \mathrm{e}$ & -4.99 & $9 \%$ metal, $8 \%$ trans- $\mathrm{CN}, 80 \%$ cis- $\mathrm{CN}$ \\
\hline $6 b_{1}$ & -5.08 & $100 \%$ cis- $\mathrm{CN}$ \\
\hline $2 b_{2}$ & -5.41 & $100 \%$ cis- $\mathrm{CN}$ \\
\hline $20 \mathrm{a}_{1}$ & -6.34 & $7 \%$ metal, $65 \%$ trans- $\mathrm{CN}, 27 \%$ cis- $\mathrm{CN}$ \\
\hline $5 b_{1}$ & -6.58 & $19 \%$ of metal, $80 \%$ cis-CN \\
\hline $9 \mathrm{e}$ & -6.70 & $12 \%$ of metal, $80 \%$ cis-CN \\
\hline $19 \mathrm{a}_{1}$ & -7.25 & $8 \%$ metal, $87 \%$ cis- $\mathrm{CN}$ \\
\hline $8 \mathrm{e}$ & -8.18 & $63 \%$ of metal d(xz, yz), $18 \%$ NO \\
\hline $18 \mathrm{a}_{1}$ & -9.14 & $15 \% \mathrm{Fe}, 64 \%$ trans- $\mathrm{CN}, 21 \%$ cis- $\mathrm{CN}$ \\
\hline $1 \dot{\mathrm{b}}_{2}$ & -10.23 & $92 \%$ of metal d(xy), $8 \%$ cis-CN \\
\hline
\end{tabular}

Atomic Mulliken population.

-0.25 for $\mathrm{Fe},+0.02$ for trans-C, -0.35 for trans-N, -0.06 for cis- $\mathrm{C},-0.32$ for cis- $\mathrm{N},+0.29$ for $\mathrm{N}(\mathrm{NO}),-0.13$ for $\mathrm{O}(\mathrm{NO})$.

Table 4. Ionization energies for nitroprusside calculated at SCF and CI levels with the DZSL basis set. The CI weigth refers to the following orbital list $\left\{2 \mathrm{~b}_{2} 6 \mathrm{~b}_{1} 10 \mathrm{e} 11 \mathrm{e} 1 \mathrm{a}_{2} 21 \mathrm{a}_{1} 12 \mathrm{e}\right\}$.

\begin{tabular}{lccc}
\hline State & SCF & $\mathrm{CI}(\mathrm{eV})$ & $\begin{array}{c}\text { dominant } \\
\text { configuration }\end{array}$ \\
\hline${ }^{1} \mathrm{~A}_{1}$ & -712.8984 & -713.2376 & $0.913(2244224)$ \\
${ }^{2} \mathrm{E}$ & 1.98 & 2.58 & $0.914(2244223)$ \\
${ }^{2} \mathrm{~A}_{1}$ & 3.47 & 4.45 & $0.920(2244214)$ \\
${ }^{2} \mathrm{~A}_{2}$ & 4.09 & 4.24 & $0.911(2244124)$ \\
\hline
\end{tabular}

in Koopmans' energy with the highest energy orbital of $b_{2}$ symmetry, pointed as the HOMO by SCCC calculations, lying far from the HOMO-LUMO threshold by about $5.4 \mathrm{eV}$. The HOMO shows dominant contributions from equatorial and trans $C N$ bonds in agreement with INDO results and the first LUMO, 13e, shows an intense mixture between $3 d(x z, y z)$ and the dominant $\pi^{*}$ NO contribution. The next two orbitals have a dominant metallic $s$ and $p$ character with many virtual orbitals showing negative Mulliken populations, an effect known as counter-intuitive orbital mixing $(\mathrm{CIOM})^{28}$.
Table 5. Singlet excitation energies calculated at SCF/CI levels with the DZSL basis set. The CI weigths and configurations refer to the following orbital list $\left\{2 \mathrm{~b}_{2} 6 \mathrm{~b}_{1} 10 \mathrm{e} 11 \mathrm{e} 1 \mathrm{a}_{2} 21 \mathrm{a}_{1} 12 \mathrm{e} / 13 \mathrm{e}\right\}$.

\begin{tabular}{lccc}
\hline State & SCF & CI & $\begin{array}{c}\text { dominant } \\
\text { configuration }\end{array}$ \\
\hline $\mathrm{X}^{1} \mathrm{~A}_{1}$ & -712.8984 & -713.2376 & $0.913(2244224 / 0)$ \\
$\mathrm{a}^{1} \mathrm{E}$ & - & 2.8 & $0.929(2244214 / 1)$ \\
$\mathrm{b}{ }^{1} \mathrm{E}$ & - & 3.1 & $0.917(2244124 / 1)$ \\
$\mathrm{a}^{1} \mathrm{~B}_{2}(\mathrm{xy})^{*}$ & - & 3.2 & $0.932(2244223 / 1)$ \\
\hline
\end{tabular}

* other components of this excitation have been calculated but they suffer from balance problems in CI calculation (see text).

Table 6. Excitation energies calculated at SCF and CI levels with the DZSL basis for the triplet spin states. The configurations refer to the following orbital list $\left\{2 \mathrm{~b}_{2} 6 \mathrm{~b}_{1} 10 \mathrm{e} 11 \mathrm{e} 1 \mathrm{a}_{2} 21 \mathrm{a}_{1} 12 \mathrm{e} / 13 \mathrm{e}\right\}$.

\begin{tabular}{lccc}
\hline State & SCF & CI & $\begin{array}{c}\text { dominant } \\
\text { configuration }\end{array}$ \\
\hline $\mathrm{X}^{1} \mathrm{~A}_{1}$ & -712.8984 & -713.2376 & $0.913(2244224 / 0)$ \\
$\mathrm{A}^{3} \mathrm{E}$ & 0.9 & 2.8 & $0.929(2244214 / 1)$ \\
$\mathrm{B}{ }^{3} \mathrm{E}$ & 1.9 & 3.1 & $0.917(2244124 / 1)$ \\
$\mathrm{A}^{3} \mathrm{~B}_{2}(\mathrm{xy})^{*}$ & 0.03 & 3.06 & $0.930(2244223 / 1)$ \\
\hline
\end{tabular}

* other components of this excitation have been calculated but they suffer from balance problems in CI calculation (See text). The SCF results are expected to underestimate the singlet-triplet excitation energies.

The MNSL calculation, although showing minor interchanges $\left(11 e / 1 a_{2}\right.$ and $\left.20 a_{1} / 5 b_{1}\right)$, confirmed the previous energy level ordering. These changes occur due the ligand character of these orbitals and its dependence on the basis set. Among the virtual orbitals minor differences were found, most of them, due to the limited variational freedom in MNSL calculation. However there is no chance, within the ab initio methodology, for the configuration or the nature of the frontier orbitals to be qualitatively changed due to the large energies involved.

At this point it is important to remark that the close proximity between these energy levels makes one-electron excitation assignments of minor use, as already pointed out by Calabrese and Hayes ${ }^{5}$. Such case requires an explicit calculation of many-body interactions and relaxation effects through SCF/CI calculations in order to provide reliable transition assignments. How dependent these excitation energies are on the ECP quality, basis set and electron correlation treatment must be well understood since the orbital scheme is dependent on these technicalities. To check this point out two calculations were carried out without ECP but still keeping the same basis set $(1 s, 2 s$ and $2 p$ functions were added in order to replace the ECP). Both calculations, MNSL and DZSL, generated similar orbital ordering with the metal $d$ orbitals deep in core and 
the highest $b_{2}$ orbital few eV below the HOMO-LUMO threshold. Note that in both calculations the metal basis set contained the same three $3 d$ functions! However when the metal basis was replaced, in another calculation, by a minimal one with just a single $d$ function the results turned to be completely different with the metal $d$ functions appearing as the HOMO! The orbital interpretation resulting from this calculation shows the same pattern as SCCC calculations. The lack of variational freedom in the metal $d$ orbital seems to push it to be the HOMO. Therefore an extended and balanced basis set is very important to achieve reliable results in these calculations. This seems to be clearly valid in $a b$ initio calculations and suggest how reliable minimal basis based semi-empirical (or $a b$ initio!) calculations could be in this situation. However the picture is not completely clear since recently Dr. Estrin brought to our knowledge that DFT calculations ${ }^{10}$ that support the HOMO orbital as a mixture in which the metal $d$ orbital have a dominant contribution. Some tests have been performed, like the use of the same geometry and basis set, but the results seem to indicate no basis set dependence on these calculations. The energy levels close to the HOMOLUMO threshold are similarly packed. Considering the excellent geometries and vibrational frequencies generated by this method work still have to be made to understand these difficulties. Another test carried out was to run the same RHF calculations with the Hay and Wadt's ECP's. No significant difference was found between these calculations and the ones carried out in this article. This particular test have been previously reported by Pressprich et al. ${ }^{20}$ and confirm our results.

The Mulliken atomic charges confirmed well known tendencies ${ }^{10}$ showing the cyano nitrogens negatively charged and the nitrosyl nitrogen largely positively charged. It is believed these charges are responsible for the intense solvatochromic effects observed in many bands of SNP, thus influencing the nitrosyl electrophilic reactivity.

\section{Ionization potentials}

Calculations have been carried out to check the reliability of Koopmans' energy as the ionization potential (IP) of SNP. Two major errors affect the Koopmans' energy, the relaxation energy and the correlation energy. The first might be treated through a specific ion SCF calculation while the correlation energy should be estimated through some post-SCF methodology. Calculations for the first IP are presented at SCF/CI-SD level for DZSL. It should be observed that at SCF level the $12 e$ orbital appears as the HOMO followed by the $21 a_{1}$ and $1 a_{2}$ orbitals. However at CI level an intense differential correlation energy showed the $2 l a_{1}$ more stable than $l a_{2}$ due to an extra stabilization of about $0.83 \mathrm{eV}$. Considering that further calculations indicated the $21 a_{1}$ orbital to became more stable with the increasing the basis set we should expect an error around one electron-volt for the IP assignment based on the Koopmans' energy.

\section{Electronic spectra}

Concerning the vertical electronic spectrum, self consistent field calculations were carried out for all low-lying triplet states. Although there is no expectancy that singlettriplet energies would be well described at SCF level, due to the Fermi correlation energy, this run was a necessary step towards the CI calculations. Unfortunately the singlet SCF convergence proved to be too difficult to achieve with our tools and all singlet CI calculations had to be carried out with a triplet orbital set. Therefore the singlets are expected to be few milihartrees below the values here reported. In order to search all possibilities with respect to the low energy spectrum excitations from the HOMO's to the $13 e$ level were studied; $12 e \rightarrow 13 e, 21 a_{1} \rightarrow 13 e, 1 a_{2} \rightarrow$ $13 e, 11 e \rightarrow 13 e$ at $\mathrm{CI}$ level. The calculations were carried out at SCF and CI levels with both basis set but only the DZSL results will be reported since the MNSL showed fairly good qualitative agreement.

The CI calculations were carried out with all singles and doubles excitations from the ten highest orbitals to the full set of virtuals. The CI calculations employed the interacting space approximation where just the nonvanishing spin couplings with the reference were used. Use was made of GAMESS $^{29}$ program. The number of spin-coupled configurations (CSF's) goes from 25661 CSF's in the MNSL calculation for the ground state to 70877 CSF's in an equivalent calculation with DZSL basis set. At this point it is important to remark that under the approximations employed, interacting space in CI calculations, excited states with a clear multi-reference character, our limited basis set and the ECP's use this work should be considered as a prospective $a b$ initio calculation on the vertical spectrum of this system. Further calculations are been carried out in order to provide accurate results for the vertical spectra of SNP. These employ extended basis sets, MC-SCF wave function and multi-reference CI (MRCI) calculations. The results so far confirm the present ones.

Even if the HOMO is the $12 e$ orbital, the first excited state results from $21 a_{1} \rightarrow 13 e$ excitation and have a transition energy of $2.8 \mathrm{eV}$ at CI-SD level. The polarization pattern is consistent with band I being perpendicularly polarized to the NC-Fe-NO plane. The second excitation, $1 a_{2} \rightarrow 13 e$, shows the same final symmetry, E, and was placed by CI calculations at $3.1 \mathrm{eV}$ with a perpendicular polarization as well. The SCF excitation energies seem to be systematically smaller than the experimental values due to the well-known tendency of the ROHF method to under-estimate singlet-triplet excitation energies and to the symmetry breaking observed for the excited states. Althougth SCF excitation energies with symmetry broken 
wavefunctions are often underestimated the CI results usuallly offer reliablle results for the excitation energies. However our final results rely on CI calculations that usually show a reasonable estimative of this property. In both calculations the $\mathrm{CI}$ roots are represented by the corresponding SCF dominant determinant slightly perturbed by single and double excitations. Further calculations, not reported here, indicate the intense dependence of the $a_{2}$ energy level with the ligand basis set. Such dependence makes this assignment possibly interchanged with respect to the $21 a_{1}$ $\rightarrow 13 e$ transition as already pointed out based on the differential correlation energy in the IP calculations. Both transitions appear with small oscillator strengths, similar polarization pattern and may share together a common envelope within band I.

The next band comes from the $12 e \rightarrow 13 e$ excitation and expands four different components, $B_{2}$ ( $x y$ and $y x$ ), $B_{1}\left(x^{2}-y^{2}\right)$ and $A_{l}\left(x^{2}+y^{2}\right)$. The $B_{2}(x y$ or $y x)$ is well described by a single-reference SCF wave function, however the two others, $B_{l}\left(x^{2}-y^{2}\right)$ and $A_{l}\left(x^{2}+y^{2}\right)$, require at least a two-determinant wave function in the triplet spin case. Therefore we decided to run simple ROHF/CI-SD calculations for the ${ }^{3} \mathrm{~B}_{2}$ state and use its orbitals to carry out the singlet CI calculation. The transition energy obtained, $3.1 \mathrm{eV}$, seems to provide an accurate estimate of the $12 e \rightarrow 13 e$ excitation in good agreement with Bottomley and Grein's results. The problem remain on the two other states since they correspond to higher roots in the CI calculation of the fundamental state and cannot be calculated at SCF level neither can be ensured that the configuration list actually represent single and doubles excitation from the dominant configuration, due to the single-reference character of our excitation program. Its CI results, not presented, are too high due to the differential excitation treatment with respect to the fundamental state. As an alternative we presented restricted CAS-SCF results in which most of the orbitals but the $(12 e, 13 e)$ active space were freezed. Calculations were carried out for the fundamental and the excited states and the results point to an excitation energy of about $5.5 \mathrm{eV}$ for the ${ }^{1} B_{l}\left(x^{2}-y^{2}\right)$ and ${ }^{1} A_{l}\left(x^{2}+y^{2}\right)$ and $4.89 \mathrm{eV}$ for the ${ }^{1} B_{2}(x y)$ state. Considering the $\mathrm{CI}$ result for the first term of this multiplet, $3.1 \mathrm{eV}$, and the $0.6 \mathrm{eV}$ value reported by Grein as the splitting of this multiplet, our restricted CAS-SCF result is probably still high by one electron-volt and should get smaller with a full CAS-SCF and a proper MR-CI calculation. However this result helps assigning transitions with parallel polarization pattern between 3.1 and $3.5 \mathrm{eV}$ in agreement with the experimental results. Table 7 presents the final conclusions to our calculations with the orbital assignments and the excitation energies.

Despite the Mulliken analysis for the ground state suggests an increasing of metal atomic population along $21 a_{1}$ $\rightarrow 13 e, 1 a_{2} \rightarrow 13 e$, and $12 e \rightarrow 13 e$ excitations, SCF
Table 7. Excitation energies and our proposed assignments for the eletronic spectra of SNP.

\begin{tabular}{lccc}
\hline Band & energy $(\mathrm{eV})$ & our results & polarization \\
\hline $\mathrm{I}$ & 2.8 & $21 \mathrm{a}_{1} \rightarrow 13 \mathrm{e}$ & $\perp$ \\
$\mathrm{I}$ & 3.1 & $1 \mathrm{a}_{2} \rightarrow 13 \mathrm{e}$ & $\perp$ \\
II & 3.2 & $12 \mathrm{e} \rightarrow 13 \mathrm{e}\left({ }^{1} \mathrm{~B}_{2} ; \mathrm{xy}\right)$ & || \\
$\mathrm{IV}(\mathrm{CT})$ & 4.5 & $8 \mathrm{e}\left(\mathrm{d}_{\mathrm{xz}, \mathrm{yz}}\right) \rightarrow 13 \mathrm{e}$ & || \\
$\mathrm{V}(\mathrm{CT})$ & 5.0 & $\begin{array}{c}1 \mathrm{~b}_{2}\left(\mathrm{~d}_{\mathrm{xy}}\right) \rightarrow 13 \mathrm{e} \text { part of } \\
\text { band Vmall intensity }\end{array}$ & $\perp$ \\
\hline
\end{tabular}

calculations on the triplet states showed a minor metal population depletion along such excitations. The iron atomic population change $21 a_{1} \rightarrow 13 e$ excitation is the smallest estimated, -0.18 with a calculated oscillator strength (RHF level) of 0.000938 in close agreement with the low intensity profile for this transition in the optical spectrum $(\varepsilon \cong 8)$. The population analysis showed an electron flux from the trans- $\mathrm{CN}$ to $\mathrm{NO}$ and an intense relaxation in the excited state orbitals leading to the iron depletion. The following excitations, $1 a_{2} \rightarrow 13 e$, and the $12 e \rightarrow 13 e$, point out to similar changes with about -0.23 electrons for iron atomic population change. The eletronic flux are directed from cis-CN and trans-CN to $N O$. The reported CT is consistent with the increasing intensity of these transitions ( $\varepsilon \cong 40$, o.s. $\cong 0.0063$ ). From the studied states just the $1 a_{2} \rightarrow 22 a_{1}$ excitations reported a large increase on the iron atomic population. Based on the reported values none of the first bands in the UV-visible spectrum could be assigned to CT.

Approximate calculations for the metal $\rightarrow \mathrm{NO} \mathrm{CT}$ bands were carried out by estimating the differential correlation energy for the active orbitals only. The results assign the $8 e \rightarrow 13 e$ orbital excitation, with a calculated energy transition of $4.52 \mathrm{eV}$ at CI level, to be the band IV in fairly good agreement with the experimental CT band at $4.6 \mathrm{eV}$. The next band, V, has similar energy as the second component for the $d \rightarrow N O$ transition and was calculated at CI level to lye at $5.04 \mathrm{eV}$ above the ground state. Calculations of the oscillator strengths for these transitions confirmed the first one as band IV but showed the second to have a fairly small intensity to be considered band V. At this point is clear that metal excitations from $b_{2}$ orbitals to axial ligands would not lead to intense transitions in the low energy region, contrary to what have been discussed in previous articles. From group theory, excitations from metallic $b_{2}$ orbitals are allowed ( $C_{4 v}$ point group) just to $b$ and $e$ orbitals. Excitations from atomic to ligand $d_{\mathrm{xy}}$ orbitals would lead to intense bands but its threshold of appearance is around 10-12 eV for NO. For the $b_{2} \rightarrow e_{y}$ excitation, it must be polarized along $x$ to allow excitation from $b$ to $e_{\mathrm{y}}$ 
and along y during $b_{2} \rightarrow e_{x}$ excitations leading to small oscillator strengths. This is particularly important since early articles wrongly suggested the unusual CT small intensity as an experimental evidence against the Manoharan and Gray assignment. Actually the excitation from atomic $b_{2}$ to axial nitrosyl ligand orbitals of e symmetry in $C_{4 v}$ point group, is a untypical CT band for which the main polarization is forbidden. Such "invisibility" does not violate our arguments concerning its inner-valence character. No attempts have been made toward calculating the metalto-cyano band.

The calculated triplet states follow very closely the energy ordering of the correspondent singlet. Wasielewska et al. $^{7,9}$ pointed that rather small energy differences have been calculated for singlet-triplet splitting. Our CI calculation for the $a^{3} E$ and $a^{1} E\left(21 a_{1} \rightarrow 13 e\right)$ energy difference were estimated to be 1.05 milihartrees with the triplet state more stable. The energy difference resulting from $1 a_{2} \rightarrow$ $13 e$ excitation was calculated to be 0.13 milihartrees favoring the triplet as well. Comparing these values with the average thermal energy available, $\langle\mathrm{E}\rangle=3 \mathrm{kT} / 2$, we obtain the result of 220 and $27 \mathrm{~K}$ for the temperature where both metastable states, HMS-NP and LMS-NP, would decay. Under the presented scheme both states could be reached through optical excitation with light with wavelength shorter than $540 \mathrm{~nm}$ followed by interconversion to the triplets. Although the quantitative agreement should be considered fortuitous, particularly due to opposing results coming from ESR experiments, the calculations indicate the possibility of interpreting the long-life of such states as a typical phosphorescent mechanism.

\section{Chemical Evidences}

Many experiments have been claimed to support Bottomley's views, however the most clarifying ones relates to X-ray photoelectron spectrum ${ }^{5}$, photochemical studies and a new re-interpretation of thermolysis on SNP crystals here presented.

Calabrese and Hayes ${ }^{5}$, studying the X-ray photoelectron spectrum of hexacyano metallates of chromium, manganese, iron and cobalt compared some of its features, specially the one originated from $t_{2 g}$ orbital, pointing out that such feature does not appear in the photoelectron spectrum of SNP. They emphasizes the large difference found between the hexacyano ferrate and the SNP spectrum. Another point of interest is the behavior of the $9 \mathrm{eV}$ peak, assigned to the $4 \sigma$ cyano orbital, that shifts $1.5 \mathrm{eV}$ to low energy in SNP. This offers an indication of the cyano energy levels approximation to the HOMO-LUMO threshold.

Photochemical experiments ${ }^{7,30,32}$ carried out concentrate on the estimate of the photooxidation quantum efficiency, since the behavior of this property with the light frequency is clearly related to the CT process. This study have been performed by different groups and the results indicate that band $\mathrm{I}$ is photochemically inactive, consistently with its polarization properties and the low absortivity found, in agreement with the present work. The increase in quantum yields for $\mathrm{Fe}$ (III) photoproduction (eq. 1), when irradiation was performed at $366 \mathrm{~nm}$ and $313 \mathrm{~nm}(0.34$ and 0.37 respectively), compared to the value found at $436 \mathrm{~nm}$ (0.17) was interpreted as showing the predominance of eq 1 against the alternative photoaquation reaction (Eq. 2),

$$
\begin{aligned}
& \mathrm{Fe}(\mathrm{II})(\mathrm{CN})_{5} \mathrm{NO}^{-2}+\mathrm{h} \mathrm{v} \rightarrow \mathrm{Fe}(\mathrm{III})(\mathrm{CN})_{5} \mathrm{H}_{2} \mathrm{O}^{-2}+\mathrm{NO} \\
& \mathrm{Fe}(\mathrm{II})(\mathrm{CN})_{5} \mathrm{NO}^{-2}+\mathrm{hv} \rightarrow \mathrm{Fe}(\mathrm{II})(\mathrm{CN})_{5} \mathrm{H}_{2} \mathrm{O}^{-3}+\mathrm{NO}
\end{aligned}
$$

In fact, irradiation experiments do not show only the appearance of $\mathrm{Fe}(I I I)$, but also the production of cyanogen $^{30}$; unfortunately, no quantum yields were obtained for the latter species. Besides, the main irradiations were not performed strictly at the band absorption maxima. Because of the strong coupling of the metal and the cyanide orbitals, either iron oxidation or cyanogen release could be obtained in the primary process through irradiation with energies corresponding to band II and III. However, the product yield could also be dependent on further intramolecular processes and solvatational energies associated to $\mathrm{Fe}(\mathrm{III})$ production. The quantum yields for $F e(I I I)$ production increased significantly when going from water to acetonitrile solutions ${ }^{32}$. No clear explanation of these facts have been advanced. We emphasize here on the increase in the energy of the HOMO as a function of the decrease in acceptor ability of the solvent; thus, the oxidation process becomes more favorable in acetonitrile than in aqueous solutions. We also expect that in acetonitrile medium, the composition of the HOMO will change, with a greater participation of $p$-donnor contributions of cyanides. In fact, the calculations in vacuum may be ascribed to a solvent of negligible acceptor character; thus, the presently reported results on the composition of the HOMO are in agreement with the strong intramolecular electron shifts detected in SNP when dissolved in different solvents ${ }^{10}$.

A complementary approach to the above discussion can be obtained by analyzing the stoichiometry of the thermal decomposition reaction of SNP. By heating above the temperature of water removal $\left(120^{\circ} \mathrm{C}\right)$, the weight loss and the analysis of products allow to describe the thermal process as in $\mathrm{Eq} 3^{31}$ :

$$
\begin{array}{r}
4 \mathrm{Na}_{2}\left[\mathrm{Fe}(\mathrm{CN})_{5} \mathrm{NO}\right] \rightarrow \mathrm{Na}_{4} \mathrm{Fe}(\mathrm{CN})_{6}+4 \mathrm{NO}+ \\
2 \mathrm{C}_{2} \mathrm{~N}_{2}+\mathrm{Fe}_{2}\left[\mathrm{Fe}(\mathrm{CN})_{6}\right]+4 \mathrm{NaCN}
\end{array}
$$

Interestingly, no formation of $\mathrm{Fe}(I I I)$ products was found in a deoxygenated atmosphere; instead, quantitative formation of cyanogen and $N O$ as oxidation and reduction products, respectively, showed the onset of an intramolecular CT process, with electron flow from cyanides to $\mathrm{NO}^{+}$. 
Here again, lattice effects, but no solvatational effects as in photochemical experiments, can be determinant of the different mechanistic pathway. But the results show again the importance of considering a high percentage of cyanide contribution on the HOMO. Moreover, it can be seen that the temperature for the onset of reaction 3 increase when the charge and the radius of the countercation increase and decrease, respectively, as shown by the data from several alkaline and alkaline-earth compounds. This results can be interpreted on the basis of the interactions of the cations with the free electron pairs of the cyanides. The stabilization of the electron density at cyanides makes energetically more costly the onset of reaction 3, a similar explanation as previosly given for the changes in absorption energies and redox potentials upon the change of solvent ${ }^{10}$.

\section{Conclusions}

Vertical electronic spectrum has been estimated through HF/CI-SD with an double-zeta quality orbital set. Most of the $a b$ initio results supports Bottomley and Grein's interpretations and assigns the first electronic transitions to ligand-to-ligand excitations from trans-cyano to nitrosyl ligands. The excitation energy of the two metal $\rightarrow$ $N O$ CT transitions, $8 e \rightarrow 13 e\left(d_{x z} \rightarrow \pi^{*} N O\right)$ and $1 b_{2} \rightarrow$ $13 e\left(d_{x y} \rightarrow \pi^{*} N O\right)$, have been estimated through CI calculations involving the active orbitals to be at $4.52 \mathrm{eV}$ and $5.04 \mathrm{eV}$ in remarkable agreement with the experimental results.

However some results showed to be dependent on calculation technicalities like basis, ECP or geometry and therefore many points are still open. For instance similar calculations with $\mathrm{SBK}^{23}$ basis set showed a possible interchange between the $1 a_{2}$ and $21 a_{1}$ orbitals. An important point to consider relates to the role of geometry. The slight symmetry breaking in the crystal environment would split the $12 e$ and $13 e$ levels leading to a different orbital scheme and preliminary calculations carried out confirm this suggestion. However the proper consideration of phase effects and the break of symmetry in the crystal are too far to be treated accurately with the nowadays $a b$ initio methods for the electronic structure study. Probably the major disagreement between our results and direct experimental evidence is related to the suggestion of high-spin paramagnetism upon the metastable state formation. Despite this problem there is no doubt that phosphorescent paramagnetism would easily support the existence of long-life metastable states. Other calculations must be carried out to clarify these points.

\section{Acknowledgments}

One of us $(\mathrm{EH})$ would like to acknowledge Fundação Vitae and CNPq for the financial support for this work. Dr. Estrin from Buenos Aires University are acknowledged for helpful discussions. Calculations were carried out at Laboratorio Nacional de Computação Científica (LNCC/CNPq) and acknowledgments should be done for the free computer time and the technical assistance at LNCC. JAO is a member of the research staff of the Consejo Nacional de Investigaciones Científicas y Técnicas (CONICET). All calculations used GAMESS ${ }^{29}$ program kindly offered by Dr. Mike Schmidt to LNCC. Acknowledgments to S.P.C. for the typing and the final revision.

\section{References}

1. Bottomley, F.; White, P.S. Acta Cryst. 1979, B35, 2193.

2. Manoharan, P.T.; Gray, H.B. J. Am. Chem. Soc. 1965, 87, 3340.

3. Fenske, R.F.; DeKock, R.L. Inorg. Chem. 1972, 11, 437.

4. Manoharan, P.T.; Gray, H.B. Inorg. Chem. 1966, 5, 823.

5. Calabrese, A.; Hayes, R.G. J. Amer. Chem. Soc. 1974, 96, 5054.

6. Hauser, U.; Oestreich, V.; Rohrweck, H.D. Z. Phys. 1977, A280, 17; ibid. 1977, A280, 125; ibid. 1978, A284, 9; Braga, M.; Pavão, A.C.; Leite, J.R. Phys. Rev. $B$ 1981, 23, 4328.

7. Golebiewski, A.; Wasielewska, E. J. Mol. Struc. 1980, 67, 183.

8. Bottomley, F.; Grein, F.; J.C.S. Dalton 1980, 1359.

9. Wasielewska, E. Inorg. Chim. Acta 1986, 113, 115.

10. Estrin, D.A.; Baraldo, L.M.; Slep, L.D.; Barja, C.; Olabe, J.A.; Paglieri, L.; Corongiu, G. Inorg. Chem. 1996, 35, 3897.

11. Woike, Th.; Krasser, W.; Bechtold, P.S.; Haussthl, S. Solid State Comm. 1983, 45(6), 499.

12. Krasser, W.; Woike, Th.; Bechtold, P.S.; Haussthl, S. J. Mol. Struct. 1984, 114(6), 57.

13. Krasser, W.; Woike, Th.; Haussthl, S.; Kuhl, J.; Breitschwerdt, A. J. Raman Spect. 1986, 17, 83.

14. Woike, Th.; Krasser, W.; Bechtold, P.S.; Haussthl, S. Solid State Comm. 1983, 45, 503.

15. Guida, J.A.; Piro, O.E.; Aymonino, P.J. Solid State Comm. 1986, 57, 175.

16. Guida, J.A.; Piro, O.E.; Aymonino, P.J. Solid State Comm. 1988, 68, 1007. Retzlaff, C.; Haussthl, S. Z. Kristallogr. 1987, 180, 20. Zoller, H.; Krasser, W.; Woike, Th.; Haussthl, S. Chem. Phys. Lett. 1989, 161, 497.

17. Terrile, C.; Nascimento, O.R.; Moraes, I.J.; Castellano, E.E.; Piro, O.E.; Guida, J.A.; Aymonino, P.J. Solid State Comm. 1990, 73(7), 481.

18. Woike, T.; Zollner, H.; Krasser, W.; Haussthl, S. Solid State Comm. 1990, 73, 149. Woike, T.; Haussthl, S. Solid State Comm. 1993, 86, 333. Crichton, O.; Rest, A.J. J. Chem. Soc. Dalton Trans. 1977, 10, 986. 
19.Zollner, H.; Woike, T.; Krasser, W.; Haussthl, S. Z. Kristallogr. 1989, 188, 139.

20. Pressprich, M.R.; White, M.A.; Vekhter, Y.; Coppens, P. J. Amer. Chem. Soc. 1994, 116, 5233.

21. Nakatsuji, H.; Saito, S. J. Chem. Phys. 1990, 93, 1865. Nakatsuji, H.; Saito, S. Int. J. of Quant. Chem. 1991, 39, 93.

22. Pierloot, K.; Van Praet, E.; Vanquickenborne, L.G.; Roos, B.O. J. Chem. Phys. 1993, 97, 12220.

23. Stevens, W.J.; Jasien, P.G.; Krauss, M.; Basch, H. Compact Effective Potentials for the third row atoms $K$ through $K r$, personnal communications, March, 1990. Stevens, W.J.; Krauss, M.; Basch, H. J. Chem. Phys. 1984, 81, 6026. Stevens, W.J.; Basch, H.; Krauss, M.; Jasien, P. Can. J. Chem. 1992, 70, 612. Cundari, T.R.; Stevens, W.J. J. Chem. Phys. 1993, 987, 5555.

24. Rappe, Smedley and Goddard, J. Chem. Physics 1981, 85, 2607.

25. Dunning Jr., T.H.; Hay, P.J. In Modern Theoretical Methods of Eletronic Structure Theory; Schaefer III, H.F., Ed.; Plenum Press, vol. 3, New York, 1977.

26. Huzinaga, S. J. Chem. Phys. 1965, 42, 1293.

27. Cook, D.B. Int. J. Quant. Chem. 1995, 53, 309.

28. Ammeter, J.H.; Btrgi, H.B.; Thibeault, J.C.; Hoffmann, R. J. Amer. Chem. Soc. 1978, 100(12), 3686.
29. Schmidt, M.W.; Balridge, K.K.; Boatz, J.A.; Elbert, S.T.; Gordon, M.S.; Jensen, J.H.; Koseki, S.; Matsunaga, N.; Nguyen, K.A.; Su, S.J.; Windus, T.L.; Dupuis, M.; Montgomery, J.A. J. Comp. Chem. 1993, 14, 1347.

30. Wolfe, S.K.; Swinehart, J.H. Inorg. Chem. 1975, 14, 1049. Jarzynowski, T.; Senkowski, T.; Stasicka, Z. Rocz. Chem. 1977, 51, 2299.

31. Gentil, L.A.; Olabe, J.A.; Baran, E.J.; Aymonino, P.J. Journal of Thermal Analysis 1975, 7, 279. Mohai, B.; Horvsth, A.; Honti, P.E. Journal of Thermal Analysis 1986, 31, 157.

32. Stochel, G.; van Elclik, R.; Stasicka, Z. Inorg. Chem. 1986, 25, 3663.

33. Della Vedova, C.O.; Lesk, J.H.; Varetti, E.L.; Aymonino, P.J.; Piro, O.E.; Rivero, B.E.; Castellano, E.E. J. Mol. Structure 1981, 70, 241; Rigotti, G.; Aymonino, P.J.; Varetti, E.L. J. Crystallogr. Spectrosc. Res. 1984, 14, 517; Vergara, M.M.; Varetti, E.L. J. Phys. Chem. Solids 1987, 13, 48; Amalvy, J.I.; Varetti, E.L.; Aymonino, P.J.; Castellano, E.E.; Piro, O.E.; Punte, G. J. Crystallogr. Spectrosc. Res. 1986, 16, 537; Amalvy, J.I.; Varetti, E.L.; Aymonino, P.J. An. Asoc. Quim. Argent. 1986, 74, 437. 